\title{
La valoración económica como fundamento de políticas ambientales: una revisión sistemática
}

Mgt. Rafael Fernando Vargas Salinas rfvargasv@ucvvirtual.edu.pe

Mgt. Walter Claudio Beizaga Ramírez

wbeizaga@ucvvirtual.edu.pe

Mgt. Víctor Raúl Vicente Becerra Córdova

rbecerraco14@ucvvirtual.edu.pe

Universidad César Vallejo

\section{RESUMEN}

Los métodos de valoración económica, puestos a disposición de los decisores públicos, permiten gestionar información valiosa para formular políticas públicas de carácter ambiental, ello, en un contexto de cambio climático y de necesidad de acciones ambientales sostenibles por parte del Estado y la sociedad en su conjunto. En tal sentido, el objetivo de la presente investigación es realizar una revisión sistemática de los aportes surgidos por investigaciones científicas, respecto del uso de la valoración económica en el ámbito de las decisiones públicas, así como, determinar la utilidad que reporta la valoración económica ambiental para fines de investigación y de toma de decisiones. La metodología utilizada en el presente artículo se basa en el enfoque PRISMA para revisiones sistemáticas. Se concluye que, la valoración económica aplicada a contextos ambientales, brinda información valiosa respecto del usufructo del capital natural, siendo dicha información, clave para tomar decisiones y generar políticas sostenibles, por tanto, la aplicación de la valoración económica es de gran utilidad para fines académicos como de gestión de políticas públicas ambientales.

Palabras Clave: patrimonio natural; métodos de valoración económica; decisiones públicas; políticas públicas. 


\title{
Economic valuation as a foundation for environmental policies: a systematic review
}

\begin{abstract}
The economic valuation methods, made available to public decision-makers, allow the management of valuable information to formulate public policies of an environmental nature, in a context of climate change and the need for sustainable environmental actions by the State and society in its own right. set. In this sense, the objective of this research is to carry out a systematic review of the contributions arising from scientific research, regarding the use of economic valuation in the field of public decisions, as well as to determine the utility of environmental economic valuation. for research and decisionmaking purposes. The methodology used in this article is based on the PRISMA approach for systematic reviews. It is concluded that, the economic valuation applied to environmental contexts, provides valuable information regarding the usufruct of natural capital, this information being key to make decisions and generate sustainable policies, therefore, the application of economic valuation is very useful for purposes academics and environmental public policy management.
\end{abstract}

Keywords: natural heritage; economic valuation methods; public decisions; public policies.

Artículo recibido: 10 Agosto. 2021 Aceptado para publicación: 07. Setiembre. 2021 Correspondencia: rfvargasv@ucvvirtual.edu.pe Conflictos de Interés: Ninguna que declarar 


\section{INTRODUCCIÓN}

En la última década, el planeta ha sido testigo de la imperiosa necesidad, en la que están inmersos los países en desarrollo, de diseñar e implementar políticas ambientales, no sólo para luchar contra el cambio climático (ONU, 2020) -tema aún muy discutido- sino, para generar espacios para la actuación pública, en términos de la implementación de medidas que vayan en línea con la sostenibilidad ambiental. Se ha visto, desde el ámbito normativo, un incremento en la preocupación de los actores públicos, con respecto a los problemas ambientales (PNUMA, 2015, 2018; Ministerio del Ambiente del Perú, 2015, 2016), la misma que ha aumentado en función del reconocimiento colectivo del acrecentamiento de los niveles de contaminación, la pérdida de los hábitats naturales y, con ello, del aumento de los efectos nocivos de la degradación ambiental en el bienestar humano; en tal sentido, la elaboración de políticas (Masiero et al., 2019; Valencia, 2017) pasa por analizar las diferentes herramientas de intervención pública desde una perspectiva transversal que, involucra, en lo fundamental, aspectos legales, económicos y ambientales (Galarza y Ruíz, 2017), ya que esto permite ampliar la comprensión de las herramientas disponibles para hacer frente a los problemas públicos (Dunn,2018).

La preocupación pública, se ha traducido en aspectos normativos, que se relacionan con materias específicas de protección y conservación ambiental, siendo que, en el ámbito colectivo, las medidas para luchar contra la contaminación se traducen en medidas legislativas específicas (Costanza et al., 2017). Las políticas ambientales (Ripka et al., 2018), siendo medidas deliberadas de la intervención del Estado en un ámbito específico, se señala, son necesarias debido a que el suministro de los bienes y servicios ambientales sufre de las denominadas fallas de mercado; es decir, se hace necesaria la intervención pública, ya que el mecanismo de mercado no es capaz de captar el total valor de tales bienes y servicios, debido al carácter de bienes públicos (Weimer y Vining, 2017) que conllevan; por tanto, al no existir un mercado específico para el patrimonio ambiental, es necesario recurrir a medios alternativos de medición monetaria y no monetaria, haciendo uso de técnicas de valoración económica, cuyos resultados habrán de ser evaluados en términos de su papel potencial en la toma de decisiones públicas (Howlet y Mukherjee, 2017).

El presente artículo, se ocupa del asunto referente a cómo encuadrar la metodología de la valoración económica en los procesos para tomar decisiones en el ámbito público (Díaz, 
2019). La idea se fundamenta en la necesaria introducción de procedimientos racionales para tomar decisiones que derivan en políticas públicas (Lusardi et al., 2020; Marre et al., 2016; Zárate y Villegas, 2016). Desde el terreno de la Economía, se reconoce que cada proceso de toma de decisiones públicas implica varias elecciones, las cuales, se espera sean racionales, es decir, estén basadas en información técnica verificable; por tanto, el criterio de elección racional significará hacer el mejor uso de los recursos disponibles; es decir, se elegirá la opción que tenga el costo de oportunidad más bajo o, simplemente, el valor más bajo para ser sacrificado. Consiguientemente, los costos y beneficios de cualquier intervención pública deben sopesarse en comparación con los costos y beneficios de usos alternativos de los recursos.

Existen cuestiones relevantes que el artículo pretende desentrañar respecto de la normativa vinculada a la valoración económica y su utilización en el diseño, elaboración y gestión de la política pública (Fontaine, 2015), en especial, en lo concerniente a las políticas ambientales (Cerda y García, 2018). Es interesante indagar respecto de lo siguiente: ¿habrán utilizado las autoridades públicas estimaciones, provenientes de la valoración económica, para tomar sus decisiones de política ambiental? y ¿para qué tipo de decisiones públicas han utilizado la valoración económica ambiental? Para dar respuesta a estas preguntas, se procederá con una revisión sistemática de trabajos realizados en un contexto global, aunque, los ejemplos concretos de estudios de valoración económica se ocuparán específicamente del uso de los recursos ambientales. En cuanto a la metodología, la investigación es de tipo descriptivo y exploratorio de carácter documental. La indagación busca determinar la existencia de herramientas de intervención, que técnicamente bien utilizadas, servirán para alinear el comportamiento colectivo hacia el logro de ciertos objetivos públicos, así como que éstas constituyen las reglas del juego. El uso de información proveniente de métodos de valoración económica, entendido como una de las herramientas dispuestas para efectuar una intervención debe realizarse de acuerdo con las políticas públicas; la misma que, debe permitir avanzar hacia administraciones públicas más perceptivas al cambio, al contexto de su desenvolvimiento, al reconocimiento de la diversidad de actores, a la necesidad de una ciudadanía más participativa (Kholongo et al., 2020) y a las particularidades de los problemas ambientales. 
Finalmente, es importante mencionar, que existen varios métodos disponibles que se utilizan en la valoración económica ambiental, pero difieren en la demanda de información, en la construcción de los supuestos sobre agentes económicos y el entorno físico y, en los valores que los modelos (Zanchi y Brady, 2019) -que se derivan de dichos métodos- son capaces de captar. Por lo general, se distingue entre métodos de valoración económica monetarios y no monetarios; siendo que los métodos de valoración no monetarios incluyen la función causa-efecto y la función daño generado (Cerda y Tironi, 2017). En ambos casos, se asume la existencia de una relación técnica específica entre el bien o servicio ambiental por un lado y el consumidor por otro lado. Los métodos de valoración monetaria utilizan información de preferencias reveladas, siendo que, las preferencias reveladas incluyen el método de costo de viaje, los precios hedónicos, de un lado y; de otro, la valoración contingente, siendo ésta última la técnica de preferencia declarada más popular (Bamwesigye, 2019).

\section{METODOLOGÍA}

La ruta metodológica de la investigación, permite analizar la información bibliográfica en función de los criterios establecidos en la Metodología PRISMA (Urrútia y Bonfill, 2010) para revisiones sistemáticas. Los procedimientos metodológicos indican que, la unidad de análisis está representada por el conjunto de artículos científicos arbitrados y publicados en revistas virtuales indizadas. Las revistas que proveen los artículos que son materia prima del presente artículo de revisión, están listadas en diversas bases de datos de consulta mundial.

El criterio de búsqueda temático, realizado en español, inglés y portugués, fue el relacionado con la combinación de "valoración económica" y "políticas ambientales", "economic valuation" and "environmental policies" y "valorização econômica" y "políticas ambientais"; siendo que, se aplicó la búsqueda por los tres criterios por cada base de datos analizada. El ámbito de búsqueda se circunscribió a publicaciones de carácter mundial, en función de los idiomas seleccionados y el nivel de libre accesibilidad a las publicaciones. El filtro temporal estuvo acotado a artículos publicados entre los años 2016 a 2021. Las bases de datos utilizadas en la presente revisión son: EBSCO, SCOPUS, SCIELO, Microsoft Academic y Mendeley.

Del procedimiento realizado se obtuvo un total de 558 artículos, que permitieron incluir trabajos relacionados con los distintos métodos de valoración económica, su aplicación a 
la temática ambiental y el uso de los resultados obtenidos para tomar decisiones de política pública. Se excluyen artículos que sólo aportan mediciones más no reflexiones en torno al uso de los valores hallados para fines de diseño y gestión de políticas ambientales; así mismo, se excluyen trabajos de temática contable y agregada. El resultado de la aplicación de la metodología PRISMA, permitió obtener 29 artículos que, se encuentran alineados a los objetivos de la presente revisión sistemática y, que, se constituyen en el fundamento del presente trabajo investigativo.

Posteriormente, se procede con la extracción y el análisis de la información obtenida. Por consiguiente, los 29 artículos seleccionados fueron analizados para proporcionar hallazgos de información relevante en relación con las preguntas de investigación. La viabilidad y fiabilidad de la información extraída de los de los artículos seleccionados, en buena cuenta, lo proveen los repositorios que acogen tales investigaciones. Así, la metodología permitió acceder a las investigaciones vinculadas con la temática asociada a la "valoración económica" y las "políticas ambientales". Luego de plantear filtros temporales y de acceso, se ha podido sistematizar la información según criterios que van con el origen y los aportes a la temática de la investigación, que podrán verse a continuación. La búsqueda sistemática de palabras clave, realizadas en los repositorios seleccionados, permitió recopilar información valiosa en todos los artículos. Para darle un contenido y preservar la credibilidad de los aportes, se procede a generar un orden en función de los siguientes criterios: 1. Repositorio; 2. Título del artículo; 3. Autor(es) año de publicación; 4. Origen de la publicación; 5. Referentes teóricos; 6. Metodología utilizada; 7. Fuente de obtención de información; 8. Objetivos; 9. Conclusiones.

\section{Figura 1}

Proceso de Selección PRISMA. 
-Artículos registrados en las bases de datos: EBSCO, SCOPUS, SCIELO, Microsoft Academic y Mendeley; referidos a "valoración económica" y "políticas ambientales" en los idiomas inglés, español y portugués; para el periodo del 2016 al 2021. $\mathbf{n}=\mathbf{5 5 8}$.

Índice de Índice por relación directa con la temática de investigación.

Índice por relación con los objetivos de investigación.

Índice de artículos cribados y no repetidos
- De los 558 artículos filtrados inicialmente, quedan 57 que tienen relación con la temática de la revisión sistemática propuesta.referidos a "valoración económica" y "políticas ambientales" en los idiomas inglés, español y portugués; para el periodo del 2016 al 2021.

- De los 57 artículos relacionados a la temática de investigación, quedan $\mathbf{2 9}$ que tienen relación con los objetivos de la revisión sistemática propuesta.

-Los artículos seleccionados para la presente revisión sistemática son 29.

Tal como se puede apreciar a continuación en la Tabla 1, el proceso de cribado de las investigaciones a utilizar en la revisión sistemática, permitió realizar la búsqueda en función de una combinación doble de palabras, de un lado la "valoración económica" y de otro "políticas ambientales", lo cual, complejizó el procedimiento seguido, más aún al ser realizado en los idiomas inglés, portugués y español, por cada una de las bases de datos utilizadas.

\section{Tabla 1}

Resultados de la Búsqueda y Selección en Bases de Datos.

\begin{tabular}{|c|c|c|c|c|c|c|}
\hline Repositorio & $\begin{array}{l}\text { Criterios de } \\
\text { búsqueda* }\end{array}$ & Resultados & Temática & Acceso & Objetivos & $\begin{array}{c}\text { Artículos } \\
\text { no } \\
\text { repetidos }\end{array}$ \\
\hline EBSCO & $\begin{array}{l}\text { "Valoración } \\
\text { Económica" }\end{array}$ & 67 & 10 & 10 & 5 & 5 \\
\hline SCOPUS & $\begin{array}{l}\text { "Políticas } \\
\text { Ambientales" }\end{array}$ & 136 & 13 & 13 & 8 & 8 \\
\hline SCIELO & $\begin{array}{l}\text { "Economic } \\
\text { valuation" }\end{array}$ & 4 & 4 & 4 & 2 & 2 \\
\hline $\begin{array}{l}\text { Microsoft } \\
\text { Academic }\end{array}$ & $\begin{array}{l}\text { "Environmental } \\
\text { Policies" }\end{array}$ & 245 & 18 & 18 & 7 & 7 \\
\hline \multirow[t]{2}{*}{ Mendeley } & $\begin{array}{l}\text { "Valorização } \\
\text { econômica" } \\
\text { "Políticas } \\
\text { Ambientais" }\end{array}$ & 106 & 12 & 12 & 7 & 7 \\
\hline & TOTA & 558 & 57 & 57 & 29 & 29 \\
\hline
\end{tabular}

* Se aplicó los mismos criterios de búsqueda -en español, inglés y portugués-en cada uno de los repositorios analizados. 
Los 29 artículos científicos cribados finalmente, se vinculan directamente con los objetivos planteados en la revisión sistemática; de un lado la relación de la valoración económica ambiental y las políticas públicas y, del otro, el uso de los métodos de la valoración económica y su utilidad en temas específicos de política ambiental.

\section{RESULTADOS}

La selección, revisión y posterior análisis en función de los objetivos planteados en la presente revisión sistemática, permitió generar un flujo de información muy interesante, la misma que se presenta a continuación.

De primera intención, la tabla 2, muestra la nómina de artículos seleccionados para ser analizados, siendo que, los 29 trabajos de investigación se presentan agrupados por la base de datos que los alberga, luego en función de autoría y, finalmente, por origen.

\section{Tabla 2}

Artículos Incluidos en la Revisión Sistemática.

\begin{tabular}{|c|c|c|c|}
\hline $\mathbf{N}^{\circ}$ & Repositorio & Autor(es) & País \\
\hline 1 & EBSCO & Ripka et al. (2018). & Brasil \\
\hline 2 & EBSCO & Suárez (2020). & España \\
\hline 3 & EBSCO & Droste y Bartkowski (2018). & Alemania \\
\hline 4 & EBSCO & Shapiro et al. (2019). & Países Bajos \\
\hline 5 & EBSCO & Sung-Yoon y Jungwoo (2018). & Corea del Sur \\
\hline \multirow[t]{2}{*}{6} & SCOPUS & Milon y Alvarez (2019). & Estados \\
\hline & & & Unidos \\
\hline 7 & SCOPUS & Naime et al. (2020). & Noruega \\
\hline 8 & SCOPUS & Li et al. (2018). & China \\
\hline 9 & SCOPUS & Martínez et al. (2017). & México \\
\hline 10 & SCOPUS & Vannevel y Goethals (2020). & Bélgica \\
\hline 11 & SCOPUS & Schröter et al. (2020). & Suecia \\
\hline 12 & SCOPUS & Vidal y Ruiz (2020). & España \\
\hline \multirow[t]{2}{*}{13} & SCOPUS & Hiebert y Allen (2019). & Estados \\
\hline & & & Unidos \\
\hline 14 & Mendeley & Tarupí y De la Fuente (2016). & Colombia \\
\hline 15 & Mendeley & Onofri y Nunes (2020). & Italia \\
\hline \multirow[t]{2}{*}{16} & Mendeley & Sinha et al. (2018). & Estados \\
\hline & & & Unidos \\
\hline 17 & Mendeley & Getzner et al. (2018). & Austria \\
\hline 18 & Mendeley & Louda et al. (2021). & Rep. Checa \\
\hline 19 & Mendeley & Förster et al. (2019). & Alemania \\
\hline 20 & Mendeley & Del Giudice et al. (2017). & Italia \\
\hline 21 & Microsoft Academic & Díaz et al. (2018). & Cuba \\
\hline 22 & Microsoft Academic & Ambarita et al. (2018). & Gales \\
\hline
\end{tabular}




\begin{tabular}{lcll}
23 & Microsoft Academic & Marušić et al. $(2018)$. & Croacia \\
24 & Microsoft Academic & Romero et al. $(2020)$. & Chile \\
25 & Microsoft Academic & Bartkowski et al. $(2020)$. & Alemania \\
26 & Microsoft Academic & Shao et al. $(2020)$. & China \\
27 & Microsoft Academic & Tinch et al. $(2019)$. & Bélgica \\
28 & SCIELO & Martínez y Sainz $(2017)$. & México \\
29 & SCIELO & Bardy et al. $(2016)$. & Brasil \\
\hline
\end{tabular}

\section{Tabla 3}

Distribución de Artículos por Repositorio.

\begin{tabular}{lcc}
\hline Repositorio & $\mathbf{N}^{\circ}$ de artículos & $\%$ \\
\hline EBSCO & 5 & $17 \%$ \\
SCOPUS & 8 & $28 \%$ \\
Mendeley & 7 & $24 \%$ \\
Microsoft Academic & 7 & $24 \%$ \\
SCIELO & 2 & $7 \%$ \\
\hline Total & 29 & $100 \%$ \\
\hline
\end{tabular}

\section{Figura 2}

Distribución Porcentual de artículos.

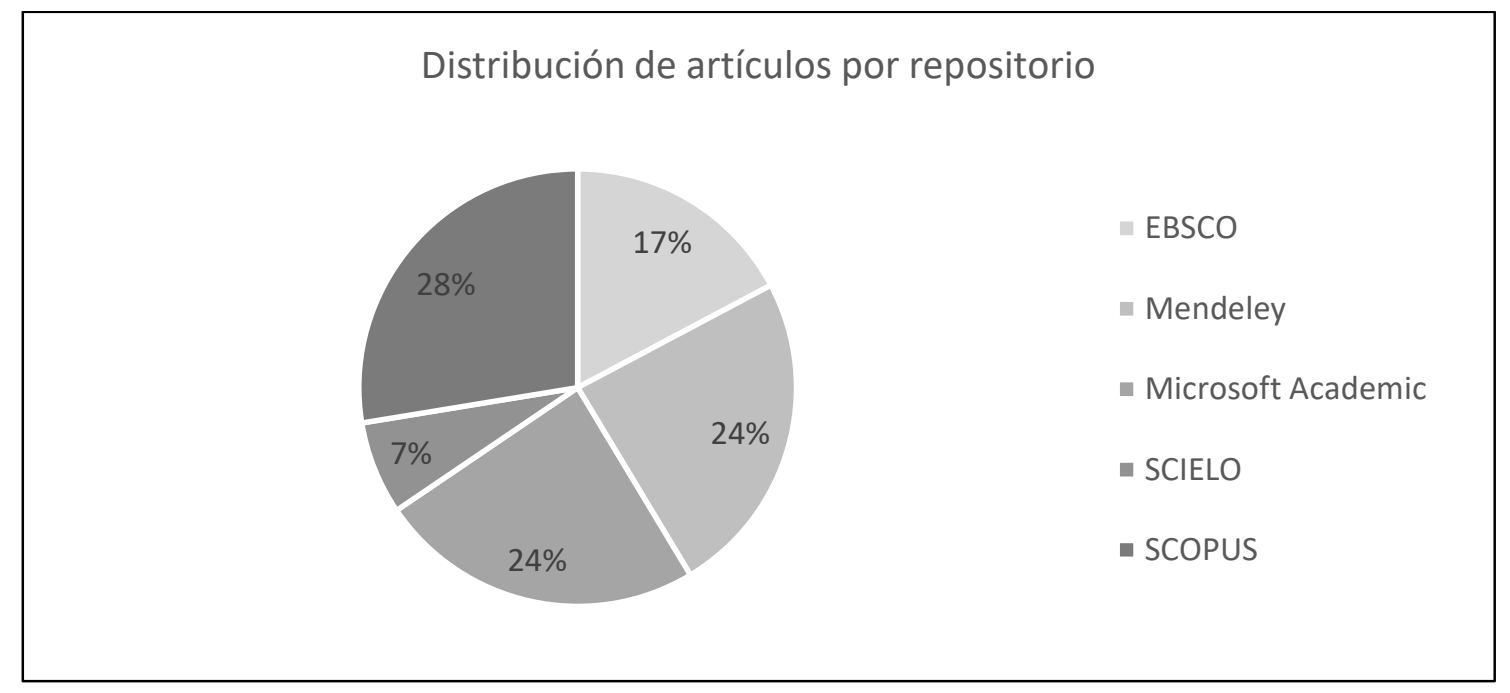

\section{Análisis de la metodología utilizada en los artículos seleccionados}

La figura 3 muestra un esquema que refleja los distintos métodos de valoración económica evidenciados en las investigaciones cribadas. De ello, se tiene que los métodos de valoración económica (Naime et al., 2020) directos, son también conocidos como, métodos basados en preferencias declaradas (Shao et al., 2020) de los cuales, el más 
utilizado, de acuerdo con la revisión presentada es el que corresponde a la aplicación del Método de la Valoración Contingente (Getzner et al., 2018; Li et al., 2018; Marušić et al., 2018; Suárez, 2020; Sung-Yoon y Jungwoo, 2018;) como una forma de estimar la Disposición a Pagar (Tarupí y De la Fuente, 2016; Shao et al., 2020).

\section{Figura 3}

Métodos de Valoración Económica.

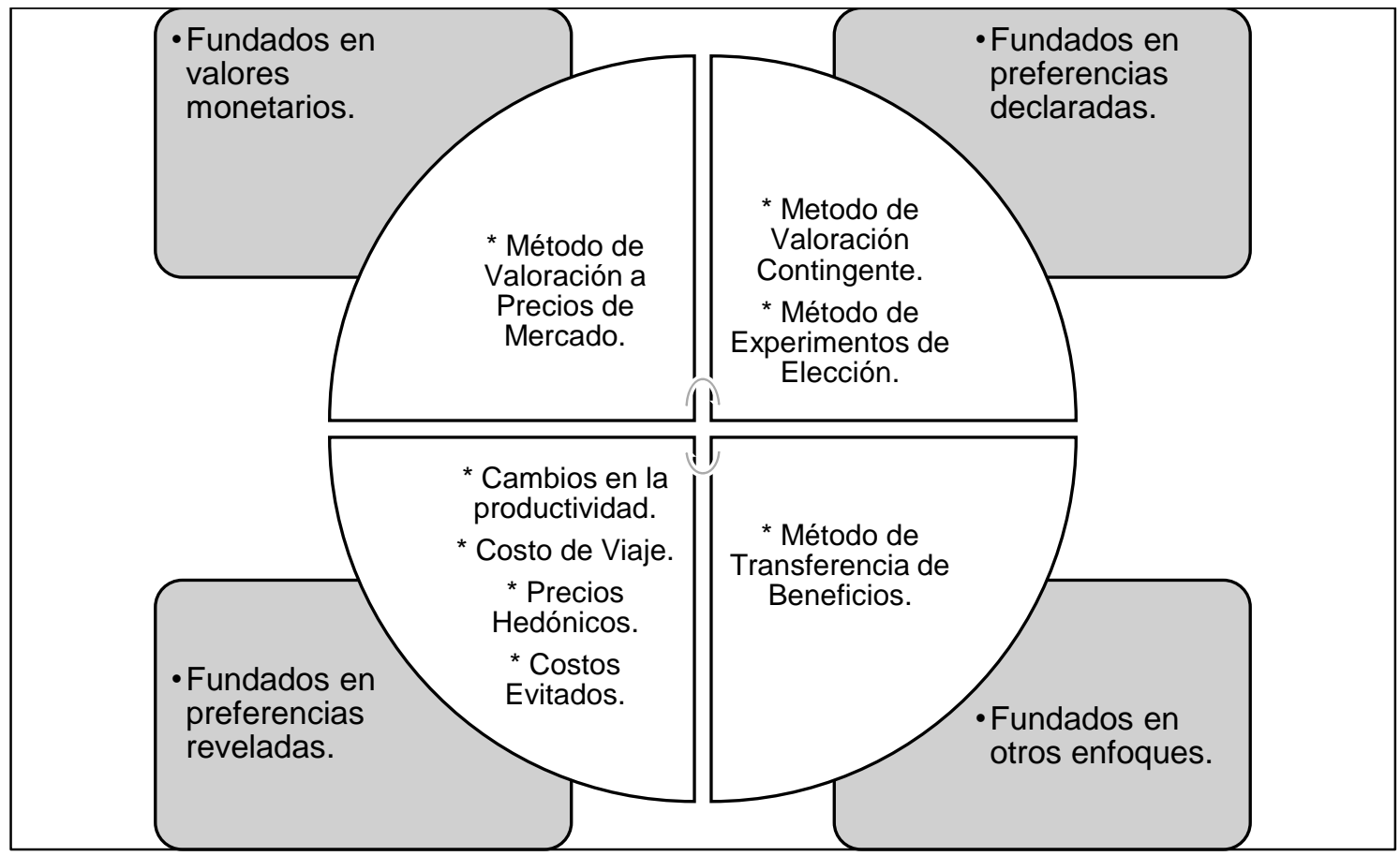

Una combinación de métodos directos e indirectos, lo representa la aplicación de los Métodos del Costo de Viaje y Valoración Contingente (Vidal y Ruiz, 2020); asimismo, la aplicación del Método del Coste de Viaje (Ambarita et al., 2018) y metaanálisis de la literatura y (Martínez y Sainz, 2017). Como métodos basados en preferencias reveladas se tiene la aplicación de un Modelo de Precios Hedónicos (Hiebert y Allen, 2019) especificado con medidas GIS (Martínez et al., 2017).

En cuanto a investigaciones de corte bibliográfico y analítico, se tienen los relacionados con el análisis, discusión conceptual y metodológica (Bardy et al., 2016; Tinch et al., 2019; Milon y Alvarez, 2019; Schröter et al., 2020; Shapiro et al., 2019; Droste y Bartkowski, 2018). Igualmente, se tienen artículos que utilizan la revisión del Estado del Arte y uso de Mapeo y Evaluación de Servicios de los Ecosistemas (Bartkowski et al., 2020). En la misma línea, se tienen los Estudios de Caso (Ripka, Da Silva y Hernández, 
2018; Del Giudice et al. 2017) y aplicación del enfoque Final Ecosystem Goods and Services (Sinha et al., 2018).

A nivel de la utilización de métodos específicos, se puede apreciar los trabajos en relación a, la aplicación metodológica para valorar económicamente los bienes y servicios ecosistémicos (BSE) y daños ambientales (Díaz et al., 2018). Luego, la identificación y análisis de estudios de valoración monetaria de los servicios ecosistémicos reguladores y culturales (Förster et al., 2019). Asimismo, la aplicación conjunta del Método del Experimento de Elección de tipo cuantitativo y el Análisis Institucional de tipo cualitativo (Louda et al., 2021). También, el análisis del Método de la Adaptación Basada en Ecosistemas (Onofri. y Nunes, 2020). Finalmente, la aplicación del Análisis Multicriterio de Valoración de Activos Ambientales (Romero et al., 2020) y del Modelo Pentatope (Vannevel y Goethals, 2020).

A continuación, se presenta en la tabla 4, el tipo de instrumento utilizado, por los autores seleccionados, para realizar su investigación. Los artículos, dependiendo se los objetivos perseguidos, muestran una preferencia a la utilización combinada de las diferentes herramientas metodológicas que posibilitan el trabajo en el ámbito de la valoración económica, resaltando el hecho del uso de fuentes primarias fundamentalmente y, en ausencia de éstas se recurre a información oficial o a una combinación de ambas. Asimismo, la revisión documental y bibliográfica, para su posterior sistematización y análisis es fundamental en los estudios seleccionados.

\section{Tabla 4}

Instrumento de recolección de datos.

\begin{tabular}{ll}
\hline \multicolumn{1}{c}{ Instrumento utilizado } & \multicolumn{1}{c}{ Autor(es) } \\
\hline Encuesta & Sung-Yoon y Jungwoo, (2018); Tarupí y De \\
& la Fuente, (2016); Getzner et al. (2018); \\
& Marušić et al. (2018); realizadas en línea \\
& (Shao et al. (2020). \\
\hline Encuesta e información secundaria & Naime et al. (2020); Li et al. (2018); Martínez \\
& et al., (2017); Del Giudice et al. (2017); Vidal \\
& y Ruiz, (2020). \\
\hline Encuesta y entrevista & Suárez., (2020); Romero et al. (2020); y, \\
& análisis institucional (Louda et al., 2021). \\
\hline Información secundaria & Díaz et al., (2018); Hiebert y Allen, (2019). \\
\hline Revisión y análisis bibliográfico & Bardy et al. (2016); Droste y Bartkowski, \\
& (2018); Förster et al. (2019); Milon y Alvarez, \\
\hline
\end{tabular}


(2019); Ripka et al., (2018); Shapiro et al. (2019); Tinch et al. (2019); y, análisis conceptual (Bartkowski et al., 2020). Y, utilización de información secundaria (Martínez y Sainz, 2017; Onofri y Nunes, 2020; Sinha et al., 2018) además, aplicación de un modelo conceptual (Vannevel y Goethals, 2020).

Gestión digital de datos Ambarita et al. (2018).

Talleres y sistematización Schröter et al. (2020).

\section{Análisis del Objetivo 1}

\section{Utilización de la valoración económica para fines de política ambiental}

El aumento poblacional en el mundo, ejerce una presión creciente sobre la provisión de los servicios ecosistémicos, la recuperación natural, la resilencia sistémica y, por ende, sobre la calidad ambiental. La dinámica demográfica ha impulsado de manera constante los procesos de expansión urbana y, con ello, la afectación de predios que anteriormente eran considerados rurales (Hiebert y Allen, 2019; Martínez et al., 2017), esta situación ha generado importantes externalidades (Del Giudice et al., 2017) que deberán ser estudiadas. Este proceso, alimentado por una creciente demanda de servicios ambientales, ha generado que los recursos ambientales comiencen a escasear y, en consecuencia, que los ecosistemas entren en un proceso de degradación, que, dependiendo de las medidas que se puedan adoptar es aún reversible. Aunque, desde distintos ámbitos existen medidas de control y gestión, la actitud de los diversos actores frente a la extracción incesante de recursos ambientales, está situación genera un amplio debate público, que involucra al gobierno, la universidad y la sociedad en su conjunto. La discusión gira en torno a, si se deben o no, implementar valoraciones monetarias (Tarupí y De la Fuente, 2016) respecto al uso y no uso de los servicios ambientales y, si la valoración obtenida es idónea (Droste y Bartkowski, 2018) para ser fuente de diseño e implementación de políticas ambientales (Ripka et al., 2018) y esquemas de pago (Akers, 2018) con criterios de sostenibilidad. El patrimonio natural y el ambiente en general, cumplen con diversas funciones (Bardy et al., 2016). que son muy valoradas por la sociedad. Por un lado, constituyen pieza indispensable de la función de producción (Sandoval et al., 2018) de un gran conjunto de bienes, que, además, de proporcionar recursos naturales cuyos servicios (Malkoc y Zauberman, 2018) son altamente demandados por los miembros de la sociedad, actúan 
como proveedor de servicios receptores de residuos. En definitiva, el sistema ambiental proporciona servicios de soporte vital para personas, incidiendo de manera directa en la comodidad ambiental (Marušić et al., 2018) y la calidad de vida de las comunidades humanas. Por consiguiente, valorar con criterios económicos el ambiente significa poder acceder a un conjunto de indicadores, entre ellos los precios de mercado y de no mercado (Martínez y Sainz, 2017), que reflejan su importancia en el bienestar de la sociedad, que, a su vez, permiten la comparación cuantitativa de alternativas a los problemas ambientales.

La aplicación de métodos de valoración económica en el ámbito de los servicios ambientales (Cerda y García, 2018; Díaz et al.,2018) resulta ser de gran interés para la colectividad en general y, para los gestores públicos en particular. La información que se obtenga de la utilización de métodos de valoración de aplicación al patrimonio natural, puede promover políticas ambientales más sostenibles. Por tanto, estos instrumentos de valoración pueden proporcionar nuevos conocimientos (Milon y Alvarez, 2019) y evidencia útil para respaldar tales políticas, cuantificando el valor económico asociado con el uso y la protección de tales recursos (Costanza, 2020). Sin embargo, aunque existe un potencial (Bartkowski et al., 2020) para la generación de información, también existe una dificultad inherente en la implementación de métodos para evaluar la valoración económica del patrimonio natural, principalmente como resultado de la ausencia de un mercado en específico y, por tanto, un precio que explicite su demanda social.

Es importante señalar que, la valoración económica no significa establecer una valoración monetaria vinculante o generar directamente un precio de mercado. Al relacionar una cantidad monetaria con el valor económico de un servicio ambiental, no se intenta asignar un precio, sino, contar con un simple indicador monetario (Romero et al., 2020) del valor que tiene para un individuo o un colectivo de individuos (Bamwesigye, 2019). En otras palabras, lo que se pretende es reflejar de alguna manera lo que se sacrifica o a lo que las personas renuncian (Getzner et al., 2018) para mantener ese servicio ambiental. Además, dado que, en general, a lo que se renuncia es bienestar (Li et al., 2018); una forma común de medirlo es en términos de ingresos monetarios (Förster et al., 2019). Por tanto, este valor económico no significa que esa cantidad subrogará un precio de mercado, pero representa un indicador útil para fines comparativos. Se debe aclarar, que el componente que confiere valor al patrimonio natural, es su componente del valor de uso; significa que 
corresponde a la medida del bienestar que reporta al individuo y la sociedad, el consumo activo del recurso de una forma u otra.

Generalmente, los espacios naturales han sido infravalorados dada su menor rentabilidad directa puramente en términos económicos. Sin embargo, esta situación ha cambiado y, además de lo anterior, muchas actividades recreativas (Ambarita et al., 2018) y los servicios del paisaje han sido reconocidos, ya que la sociedad se ha vuelto cada vez más consciente de la necesidad de proteger el ambiente. Los ecosistemas brindan una diversidad de servicios transcendentales para el bienestar humano y el alivio de la pobreza. Los servicios de aprovisionamiento (Naime et al., 2020). son fundamentales para el bienestar de las personas, los servicios de soporte y regulación son cardinales para mantener las funciones vitales del ecosistema, brindan muchos beneficios a las personas. Además, el medio natural tiene importantes valores estéticos, educativos, culturales y espirituales y brindan oportunidades invaluables para el desenvolvimiento humano.

El objetivo de la política ambiental debiera ser el relacionar la economía, la sociedad y la toma de decisiones con acciones colectivas sostenibles (Vannevel y Goethals, 2020) para generar ambientes humanos con ecosistemas resilientes. Las prácticas actuales se centran en la combinación de niveles mínimos de protección ambiental. Un equilibrio social tiene como objetivo definir la cantidad de servicios del ecosistema que se puede obtener de la naturaleza, posibilitando el crecimiento económico y el bienestar social, sin causar daño al ecosistema. En la práctica, esto lleva al examen de las interacciones mutuas entre funcionamiento ecosistemas, disponibilidad de recursos naturales y toma de decisiones. Por tanto, es importante definir adecuadamente, políticas que provean esquemas de pago (Shapiro et al., 2019) que reflejen la disposición a pagar (Shao et al., 2020; Suárez, 2020; Sung-Yoon y Jungwoo, 2018) por un servicio ambiental o en su defecto la disposición a aceptar cambios en el usufructo de tales servicios, ello en virtud, de los cambios que sufre el ambiente producto de la interacción humana.

Estos ejemplos amplían la discusión de los flujos de recursos a las políticas y la gestión. También conducen a la idea básica de políticas activas con el objetivo de salvaguardar la biodiversidad y los servicios ambientales, mediante la reducción de la extracción de recursos y la contaminación, para valorar ecosistemas y riqueza natural. Las políticas deben servir para abordar los problemas ambientales aplicando estrategias de gobernanza (Louda et al., 2021) multinivel y, con ello, asegurar el acceso y la asignación de recursos. 
Esta situación indica que la sostenibilidad ambiental deba estar sujeta a cuantificación y valoración, como fundamento decisional (Lusardi et al., 2020).

Finalmente, los recursos naturales dependen de la preferencia del consumidor y de la percepción de los cambios en el bienestar. a través de impactos en los ecosistemas. Los diferentes métodos de valoración, tienen la misión de brindar información, que más allá de lo académico, sea fundamento para estimar los costos y beneficios de las intervenciones públicas, ello, aportando indicadores simples pero rigurosos (Onofri y Nunes, 2020) para tomar decisiones (Schröter et al., 2020). Todo ello significa, conocimiento teórico y conceptual (Tinch et al., 2019), sensibilización colectiva frente a los cambios en la disposición de recursos y servicios ambientales y, voluntad política para actuar en consecuencia. Entonces, deberá quedar plasmada colectivamente la idea de que los humanos dependen de la provisión de servicios ambientales y de los empeños por mejorar la gestión ambiental (Sinha et al., 2018). Asimismo, la cuantificación necesaria de la disponibilidad temporal (Vidal y Ruiz, 2020) y los usos de los servicios ambientales, que es el dominio de la contabilidad ambiental y estadísticas, habrá de estar presente en todos los escenarios de planificación gubernamental. Adicionalmente, es imprescindible, definir el uso sostenible de los recursos ambientales, significa ello, establecer límites al uso de los recursos y sus servicios, por un lado y, estimar los impactos ambientales por uso y/o no conservación, por otro lado.

\section{Análisis del Objetivo 2}

\section{Utilidad de la Valoración Económica y Política Ambiental}

$\mathrm{Al}$ respecto, se ha podido determinar un conjunto de características que le confieren utilidad a la valoración económica, para fines de diseño e implementación de políticas públicas ambientales. Tal utilidad se ve reflejada en la tabla 5, en la cual, se agrupan los trabajos por autores y en función del criterio de utilidad reportado por cada una de las investigaciones cribadas. Es importante mencionar, que las distintas investigaciones, reportan tangencialmente, en algunos casos, más de una característica asociada a la utilidad que reporta la valoración económica, que, para el caso, se ha considerado la utilidad principal reportada.

\section{Tabla 5}

Utilidad de la Valoración Económica Ambiental 
Coadyuva a incrementar la conciencia ambiental.
Ambarita et al. (2018); Getzner et al. (2018); Hiebert y Allen (2019); Marušić et al. (2018); Romero et al. (2020); Tarupí y De la Fuente (2016); Tinch et al. (2019).
Sung-Yoon y Jungwoo (2018).

Posibilita la planificación territorial y el diseño Bardy et al. (2016); Bartkowski et al. de políticas ambientales. (2019); Hiebert y Allen (2019); Louda et al. (2021); Martínez et al. (2017); Milon y Alvarez (2019); Ripka et al. (2018); Shapiro et al. (2019); Suárez (2020); Sung-Yoon y Jungwoo (2018).

Alienta la regulación ambiental. Tarupí y De la Fuente, (2016); Vannevel y Goethals (2020).

Genera mecanismos de financiamiento. Del Giudice et al. (2017); Droste y Bartkowski (2018); Li et al. (2018); Marušić et al. (2018); Onofri y Nunes (2020); Romero et al. (2020); Shapiro et al. (2019).

Sirve de insumo para la Contabilidad Nacional. Schröter et al. (2020).

Contribuye con la replicación de experiencias Shao et al. (2020); Shapiro et al. (2019). exitosas.

Permite asignar valor monetario a bienes y servicios de mercado y de no mercado.

Genera insumos para la discusión teórica y metodológica.
Förster et al. (2019); Martínez y Sainz (2017); Martínez et al. (2017); Suárez (2020).

Naime et al. (2020); Ripka et al. (2018); Schröter et al. (2020); Sinha et al. (2018); Tinch et al. (2019); Vidal y Ruiz (2020).

En definitiva, la figura 4, nos condensa la utilidad de la valoración económica en un esquema que refleja el vínculo entre el patrimonio natural y, su necesaria valoración cuantitativa en términos de los bienes y servicios que proveen los ecosistemas.

\section{Figura 4}

\section{Utilidad de la Valoración Económica Ambiental}




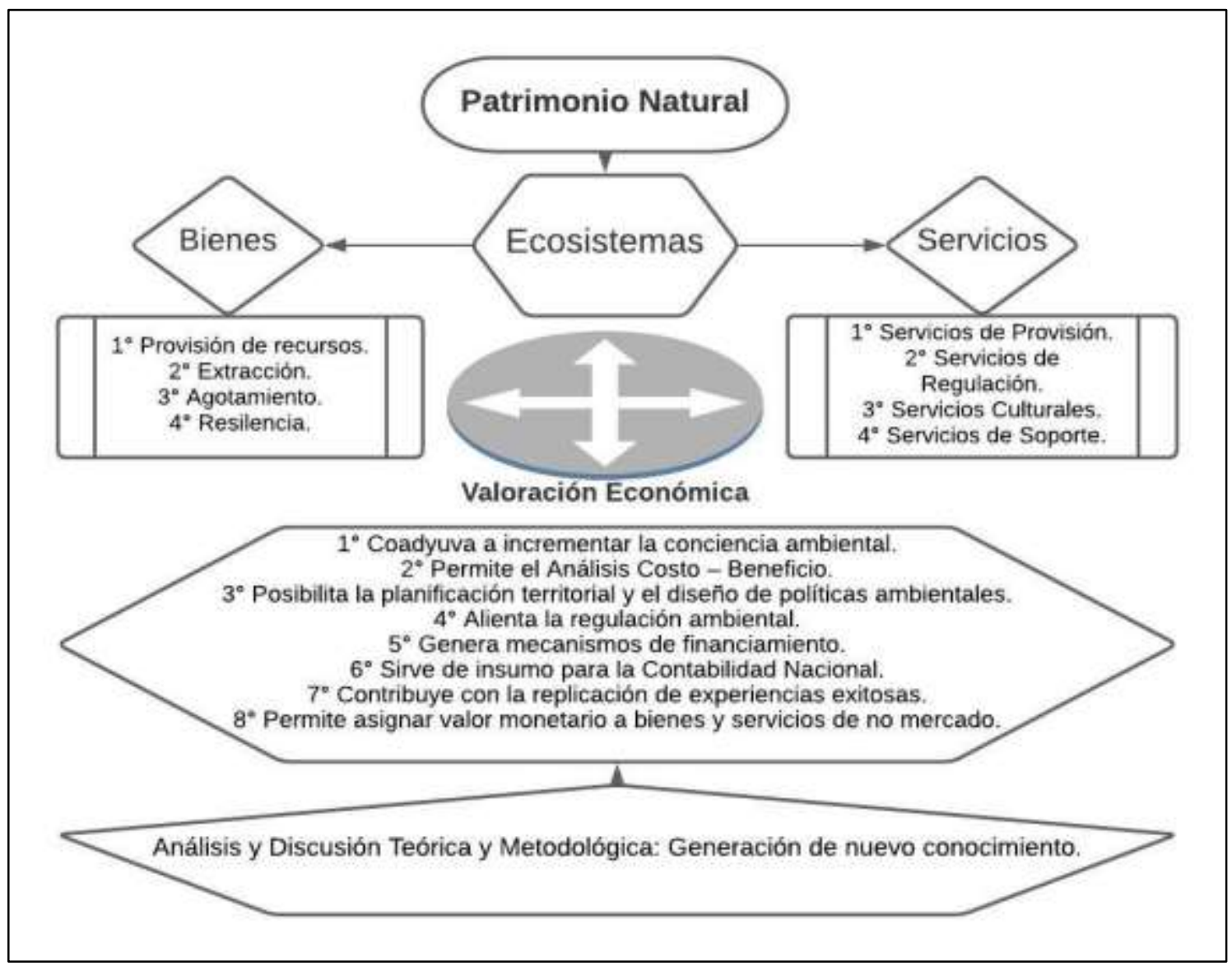

Finalmente, la valoración económica, traducida en información valiosa para los investigadores y tomadores de decisión, se convierte en una suma agregada de conocimientos que posibilitan la conservación del medio natural y permiten ponerlo al servicio del bienestar presente y futuro de la humanidad.

\section{CONCLUSIONES}

La utilidad de valorar en términos económicos los servicios ecosistémicos que brinda el patrimonio natural, radica en que el conjunto de métodos de valoración permite establecer el valor monetario y social que reporta el capital natural, captando, asimismo, la sensibilidad de quienes utilizan los bienes y servicios ambientales; es por tal razón, que esta metodología es ampliamente recomendada, para sostener técnicamente las decisiones en materia de política pública. Se ha podido determinar que los métodos de valoración aportan a la generación de procedimientos técnicos para validar la existencia de disposición a pagar (DAP) por los servicios ambientales recibidos; esto debido a que, la valoración de los ecosistemas permite tasar la disposición a pagar por mejoras ambientales; por tanto, se puede determinar el beneficio obtenido por el mejoramiento en el suministro de un determinado bien o servicio. 
La utilidad que reportan los métodos de valoración económica aplicados a temas ambientales, termina siendo fundamental para el diseño de políticas ambientales sostenibles, que se encuentren enfocadas en las generaciones presentes como en las venideras. Existen un conjunto de beneficios que se desprenden del uso de los servicios ecosistémicos, que aportan al bienestar de la colectividad en general y, que, recogidos en trabajos de investigación, no sólo posibilitan su sistematización para efectos académicos, sino, que más bien, pueden y deben ser utilizados para fines de gestión ambiental, tanto privada como pública y, siempre con criterios de sostenibilidad intergeneracional.

\section{REFERENCIAS BIBLIOGRÁFICAS}

Akers, J. (2018). Motivational Crowding in Payments for Ecosystem Service Schemes: a Global Systematic Review. Conservation and Society (V.17, 4). Recuperado de: https://www.jstor.org/stable/26869208

Ambarita, S., Basyuni, M., Sulistyono, N., Wati, R., Fitri, A., Slamet, B., Balke, T., Bunting, P. y Munir, E. (2018). Landscape planning and economic valuation of mangrove ecotourism using GIS and Google Earth image. Journal of Theoretical and Applied Information Technology, 96(19). ISSN: 1992-8645 www.jatit.org

Bamwesigye, D. (2019). Expressed Preference Methods of Environmental Valuation: Non-Market Resource Valuation Tools. Journal of Landscape Management, 10 (1). DOI: 10.20944/preprints201907.0116.v1

Bardy, R., Cardoso, E., Guimarães, J. Eufrausino, A., Machado, F., Ruiz, J., Pacobayba, A., Moreira, J., Carvalho, B., Carvalho, I., Reatto, A., Parron, L., Clemente, E., Kangussu, G., Dias, A. y Simões, M. (2016). Current overview and potential applications of the soil ecosystem services approach in Brazil. Pesquisa Agropecuária Brasileira, Brasília, v.51, n.9, p.1021-1038. DOI: 10.1590/S0100204X2016000900002

Bartkowski, B., Bartke, S., Helming, K., Paul, C., Techen, A. y Hansjürgens, B. (2020). Potential of the economic valuation of soil-based ecosystem services to inform sustainable soil management and policy. PeerJ. DOI 10.7717/peerj.8749

Cerda, A. y García, L. (2018). Valoración económica del ambiente. Revista Interamericana de Ambiente y Turismo 15(1). DOI: 10.4067/S0718235X2019000100001 
Cerda, C. y Tironi, A. (2017). La evaluación no monetaria de los servicios ecosistémicos: Perspectivas para la gestión sostenible del territorio. Revista Luna Azul, núm. 45. DOI: $10.17151 /$ luaz.2017.45.17

Costanza, R. (2020). Valuing natural capital and ecosystem services toward the goals of efficiency, fairness, and sustainability. Ecosystem Services 43:101096. DOI: 10.1016/j.ecoser.2020.101096

Costanza, R., De Groot, R., Braat, L., Kubiszewski, I., Fioramonti, L., Sutton, P., Farber, S. y Grasso, M., (2017). Twenty years of ecosystem services: How far have we come and how far do we still need to go? Ecosystem Services Vol. 28: 1-16. https://doi.org/10.1016/j.ecoser.2017.09.008

Del Giudice, V., De Paola, P., Manganelli, B. y Forte, F. (2017). The Monetary Valuation of Environmental Externalities through the Analysis of Real Estate Prices. Sustainability, 9, 229; doi:10.3390/su9020229

Díaz, C. (2019). Valoración contingente en áreas protegidas: Caso Sector Amazónico, Ecuador. Revista de Ciencias Humanas y Sociales, 35(89-2), 633-657. //produccioncientificaluz.org/index.php/opcion/article/view/27503

Díaz, D., Gonzáles, Y., Pérez, R. y Reyes, A. (2018). Valoración económica de bienes y servicios ecosistémicos. caso: Reserva Ecológica Bahía Nuevas Grandes la Isleta. Manati. Las Tunas. Revista Académica de Investigación Tlatemoani, 27. www.eumed.net/rev/tlatemoani/index.htm

Droste, N. y Bartkowski, B. (2018). Ecosystem service valuation for national accounting: a reply to Obst, Hein and Edens (2016). Environmental and Resource Economics 71:205-215 https://doi.org/10.1007/s10640-017-0146-3

Dunn, W. (2018). Public Policy Analysis: An Integrated Approach. Routledge, Sixth Edition - New York. https://es.b-ok.lat/book/4974869/6175bc

Fontaine, G. (2015). El análisis de políticas públicas: conceptos, teorías y métodos. Barcelona, España/Quito, Ecuador: Anthropos Editorial/ FLACSO.

Förster, J., Schmidt, S., Bartkowski, B., Lienhoop, N., Albert, C. y Wittmer, H. (2019). Incorporating environmental costs of ecosystem service loss in political decision making: A synthesis of monetary values for Germany. PLOS ONE 14(2): e0211419. https://doi.org/10.1371/journal.pone.0211419 
Galarza, E. y Ruíz, J. (2017). Identificación de instrumentos económicos, financieros, regulatorios y fiscales implementados por el Gobierno del Perú para un enfoque integrado del cambio climático. Publicación de las Naciones Unidas LC/TS.2017/56.

Recuperado

de: https://repositorio.cepal.org/bitstream/handle/11362/42057/S1700557_es.pdf?se quence $=1 \&$ isAllowed $=\mathrm{y}$

Getzner, M., Meyerhoff, J. y Schläpfer (2018). Willingness to Pay for Nature Conservation Policies in State-Owned Forests: An Austrian Case Study. Forests, 9, 537; doi:10.3390/f9090537

Hiebert, J. y Allen, K. (2019). Valuing Environmental Amenities across Space: A Geographically Weighted Regression of Housing Preferences in Greenville County, SC. Land, 8, 147; doi:10.3390/land8100147

Howlet, M. y Mukherjee, I. (2017). Policy design: From tools to patches. Canadian public administration. Administration publique $d u$ canada, 60(1) 140-144. https://doi.org/10.1111/capa.12209

Kholongo, W.; Lambert, E.; Kaunda, E.; Katengeza, S.; Malunga, A. (2020). Key Factors That Influence Sustainability of Community Based Advocacy Groups After Phaseout of Donor Support. International Journal of Sustainable Development Research. 6, 73-79. doi: 10.11648/j.ijsdr.20200604.13

Li, H., Yang, X., Zhang, X., Liu, Y. y Zhang, K. (2018). Estimation of Rural Households' Willingness to Accept Two PES Programs and Their Service Valuation in the Miyun Reservoir Catchment, China. Sustainability, 10, 170; doi:10.3390/su10010170

Louda, J., Vojácek, O. y Slavíková, L. (2021). Achieving Robust and Socially Acceptable Environmental Policy Recommendations: Lessons from Combining the Choice Experiment Method and Institutional Analysis Focused on Cultural Ecosystem Services. Forests, 12, 484. https://doi.org/10.3390/f12040484

Lusardi, J., Sunderland, T. J., Crowe, A., Jackson, B. M. y Jones, G. (2020). Can processbased modelling and economic valuation of ecosystem services inform land management policy at a catchment scale? Land Use Policy 96, 1-12. https://doi.org/10.1016/j.landusepol.2020.104636 
Malkoc, S. y Zauberman, G. (2018). Psychological analysis of consumer intertemporal decisions. Consumer Psychology Review. 97-113. DOI: 10.1002/arcp.1048

Marre, J., Thebaud, O., Pascoe, S., Jennings, S., Boncoeur, J., y Coglan, L. (2016). Is economic valuation of ecosystem services useful to decision-makers? Lessons learned from Australian coastal and marine management. Journal of Environmental Management, 52-62. http://dx.doi.org/10.1016/j.jenvman.2016.04.014

Martínez, A. y Sainz, J. (2017). El valor de dos espacios recreativos periurbanos en la Ciudad de México. El Trimestre Económico, vol. LXXXIV (4), núm. 336, pp. 805-846. https://doi.org/10.20430/ete.v84i336.607.

Martínez, E., Pérez, E. y Aguilar, A. (2017). Hedonic pricing model for the economic valuation of conservation land in Mexico city. El Trimestre Económico, vol. LXXXIV (4), núm. 336, pp. 805-846. https://doi.org/10.20430/ete.v84i336.607.

Marušić, Z., Sever, I., Basta, J. y Žmuk, B. (2018). Towards informed and responsible environmental management: A case study of economic valuation of natural resources in Croatia. Tourism. Vol. 66/ No. 4/ 2018/ 461 - 475 UDC: $338.484+338.483 .11(497.5)$

Masiero, M., Pettenella, D., Boscolo, M., Barua, S., Animon, I. y Matta, R. (2019). Valuing forest ecosystem services A training manual for planners and project developers. Forestry Working Paper, No. 11. Rome, FAO. Licence: CC BY-NCSA 3.0 IGO.

Milon, J. y Alvarez, S. (2019). Coastal Resources Economics and Ecosystem Valuation. Water, 11, 2206; doi:10.3390/w11112206

Ministerio del Ambiente del Perú. (2015). Manual de valoración económica del patrimonio natural. Dirección General de Evaluación, Valoración y Financiamiento del Patrimonio Natural. MINAM, GIZ. Lima, Perú.

Ministerio del Ambiente del Perú. (2016). Guía de valoración económica del patrimonio natural. 2da. Edición. Dirección General de Evaluación, Valoración y Financiamiento del Patrimonio Natural. MINAM, Lima. Perú.

Naime, J., Mora, F., Sánchez, M., Arreola, F. y Balvanera, P. (2020). Economic valuation of ecosystem services from secondary tropical forests: trade-offs and implications 
for policy making. Forest Ecology and Management. 473-118294. https://doi.org/10.1016/j.foreco.2020.118294

Onofri, L. y Nunes, P. (2020). Economic valuation for policy support in the context of ecosystem-based adaptation to climate change: An indicator, integrated based approach. Heliyon 6, e04650. https://doi.org/10.1016/j.heliyon.2020.e04650

ONU. (2020). The Sustainable Development Goals Report 2020. División de Estadísticas Mundiales de las Naciones Unidas. https://unstats.un.org/sdgs/report/2020/TheSustainable-Development-Goals-Report-2020.pdf

PNUMA (2015). Aportes Legislativos de América Latina y El Caribe en materia de Cambio Climático. Edit. Programa de las Naciones Unidas para el Medio Ambiente. Panamá. Recuperado de: http://www.unep.org/publications.

PNUMA (2018). Gobernanza ambiental y la Agenda 2030 Avances y buenas prácticas en América Latina y el Caribe. Edit. Programa de las Naciones Unidas para el Medio Ambiente. Panamá. Recuperado de: http://www.unep.org/publications.

Ripka, A., Da Silva, L. y Hernández, A. (2018). Métodos de valoración económica ambiental: instrumentos para el desarrollo de políticas ambientales. Universidad y Sociedad, 10(1), 134-141. Recuperado de: http://rus.ucf.edu.cu/index.php/rus

Romero, C., Arancibia, P., Améstica, L., Toledo, F. y Flores, G. (2020). Economic valuation of the eco-systemic benefits derived from the environmental asset lake Laguna Santa Elena, through the multi-criteria analysis. Brazilian Journal of Biology. doi.org/10.1590/1519-6984.216218

Sandoval, M., Almendarez-Hernández, M., Nieto, A., Troyo, E., Ortega, A., Beltrán, L. (2018). Valoración económica del consumo y producción de materias primas para la fabricación de biodiésel en Guatemala. Revista de Alimentación Contemporánea y Desarrollo regional, 52. DOI: http://dx.doi.org/10.24836/es.v28i52.581

Schröter, M., Crouzat, E., Hölting, L., Massenberg, J., Rode, J., Hanisch, M., Kabisch, N., Palliwoda, J., Priess, J., Seppelt, R. y Beckmann, M. (2020). Assumptions in ecosystem service assessments: Increasing transparency for conservation. Ambio, 50:289-300 https://doi.org/10.1007/s13280-020-01379-9 
Shao, Y., Xu, X., Jiang, L. y Crastes, R. (2020). Economic Valuation of the Renewal of Urban Streets: A Choice Experiment. Sustainability, 12, 4808; doi:10.3390/su12124808

Shapiro, E., McElwee, P., Van Hecken, G. y Corbera, E. (2019). Beyond Market Logics: Payments for Ecosystem Services as Alternative Development Practices in the Global South. Development and Change 51(1): 3-25. DOI: 10.1111/dech.12546

Sinha, P., Ringold, P., Van Houtven, G. y Krupnick, A. (2018). Using a final ecosystem goods and services approach to support policy analysis. Ecosphere. Volume (9):e02382. 10.1002/ecs2.2382

Suárez, C. (2020). Valoración económica del ecoturismo el caso del santuario de fauna y flora los flamencos (Colombia). Estudios y Perspectivas en Turismo. Volumen 29 pp.1135 - 1154. ISSN 0327-5841, ISSN-e 1851-1732. Recuperado de: https://dialnet.unirioja.es/servlet/articulo?codigo=7590695

Sung-Yoon, H. y Jungwoo, S. (2018). Economic valuation of noise pollution control policy: does the type of noise matter? Environmental Science and Pollution Research. 25:30647-30658 https://doi.org/10.1007/s11356-018-3061-4

Tarupí, E. y De la Fuente, G. (2016). La política de inclusión social, valoración y servicios ambientales del reciclaje en Bogotá, Colombia. Cooperativismo y Desarrollo, 23(107). doi: http://dx.doi. org/10.16925/co.v23i107.1253

Tinch, R., Beamont, N., Sunderland, T., Ozdemiroglu, E., Barton, D., Bowe, C., Börger, T., Burgess, P., Nigel, C., Faccioli, M., Failler, P., Gkolemi, I., Kumar, R., Longo, A., McVittie, A., Morris, J., Park, J., Ravenscroft, N. Schaafsma, M., Vause, J. y Ziv, G. (2019). Economic valuation of ecosystem goods and services: a review for decision makers. Journal of Environmental Economics and Policy. 10.1080/21606544.2019.1623083.

Urrútia, G. y Bonfill, X. (2010). Declaración PRISMA: una propuesta para mejorar la publicación de revisiones sistemáticas y metaanalisis. Medicina Clínica, 135. DOI: 10.1016/j.medcli.2010.01.015

Valencia, J. (2017). Valoración de los servicios ecosistemicos de investigación y educación como insumo para la toma de decisiones desde la perspectiva de la gestión del riesgo y el cambio climático. Luna Azul (45), 11-41. DOI: 10.17151/luaz.2017.45.3 
Vannevel, R. y Goethals, P. (2020). Identifying Ecosystem Key Factors to Support Sustainable Water Management. Sustainability, 12, 1148; doi:10.3390/su12031148

Vidal, F. y Ruiz. C. (2020). The Valuation of Recreational Use of Wetlands and the Impact of the Economic Crisis. International Journal of Environmental Research and Public Health, 17, 3228; doi:10.3390/ijerph17093228

Weimer D., y Vining A. (2017). Policy Analysis: Concepts and Practice. Nueva Jersey, USA: Prentice Hall. https://es.b-ok.lat/book/2847758/f44457

Zanchi, G. y Brady, M. (2019). Evaluating the contribution of forest ecosystem services to societal welfare through linking dynamic ecosystem modelling with economic $\begin{array}{llll}\text { valuation. } & \text { Ecosystem } & \text { Services } & 39 .\end{array}$ https://doi.org/10.1016/j.ecoser.2019.101011

Zárate, C. y Villegas, C. (2016). Estrategias para la sostenibilidad financiera de las áreas protegidas en Colombia. Revista Ensayos de Economía. No. 49. https://doi.org/10.15446/ede.v26n49.63825 